\title{
Safety and adequacy of endoscopic ultrasound-guided random liver biopsy in comparison with transjugular and percutaneous approaches
}

\section{(ㄷ)(i) $\Theta$}

\author{
Authors \\ Neil Bhogal' ${ }^{1}$ Bernadette Lamb², Benjamin Arbeiter ${ }^{2}$, Sarah Malik ${ }^{1}$, Harlan Sayles ${ }^{3}$, Audrey J. Lazenby ${ }^{4}$, Saurabh \\ Chandan ${ }^{1}$, Amaninder Dhaliwal ${ }^{1}$, Shailender Singh ${ }^{1}$, Ishfaq Bhat ${ }^{1}$
}

Institutions

1 Division of Gastroenterology \& Hepatology, University of Nebraska Medical Center, Omaha, Nebraska, United States

2 Department of Internal Medicine, University of Nebraska Medical Center, Omaha, NE

3 Department of Biostatistics, College of Public Health. University of Nebraska Medical Center, Nebraska, United States

4 Department of Pathology \& Microbiology, University of Nebraska Medical Center, Omaha, Nebraska, United States

submitted 21.5.2020

accepted after revision 7.8 .2020

\section{Bibliography}

Endoscopy International Open 2020; 08: E1850-E1854

DOI 10.1055/a-1274-9763

ISSN 2364-3722

(c) 2020. The Author(s).

This is an open access article published by Thieme under the terms of the Creative Commons Attribution-NonDerivative-NonCommercial License, permitting copying and reproduction so long as the original work is given appropriate credit. Contents may not be used for commecial purposes, or adapted, remixed, transformed or built upon. (https://creativecommons.org/licenses/by-nc-nd/4.0/)

Corresponding author

Ishfaq Bhat, MD, Assistant Professor, Internal Medicine, Associate Program Director - Gastroenterology Fellowship, Advanced Therapeutic Endoscopist, Division of Gastroenterology \& Hepatology, University of Nebraska Medical Center, Omaha, NE 68198, United States

Fax: +1-402-559-7777

ishfaq.bhat@unmc.edu

\section{ABSTRACT}

Background and study aims Endoscopic ultrasoundguided liver biopsy (EUS-LB) is an accepted technique for tissue acquisition. Traditionally, random LB has been performed with percutaneous (PC-LB) and transjugular (TJ-LB) approaches. The purpose of this study was to compare the safety profile and efficacy of EUS-LB, PC-LB, and TJ-LB.

Patients and methods $A$ retrospective analysis was performed at a tertiary academic medical center. Inclusion criteria for analysis were all adult patients who underwent EUS-LB since inception and TJ-LB/PC-LB over a 3-year span (June 2016 to June 2019). The primary outcome assessed was any adverse events. Secondary outcomes included technical success resulting in tissue acquisition and diagnostic adequacy of the sample for histologic analysis.

Results A total of 513 patients were included for analysis. There were 135 EUS-LB, 287 PC-LB, and 91 TJ-LB. The most common indication for $L B$ was abnormal liver function tests. For the primary outcome, the rate of adverse events was low with five reported $(<1 \%)$. There were two in the EUSLB group, two in the PC-LB group, and one in TJ-LB group, and this difference was not statistically significant $(P=$ 0.585 ). The technical success rate was $100 \%$ in each group. The rate of diagnostic adequacy was $100 \%$ in TJ-LB group and $99 \%$ in both EUS-LB and PC-LB groups. This difference was not statistically significant $(P=1.000)$. The most common histologic finding was non-specific changes (33.7\%) followed by non-alcoholic steatohepatitis (15.60\%). Conclusion In comparison with PC-LB and TJ-LB, EUS-LB has comparable safety profile, technical success rate, and diagnostic adequacy. EUS-LB should be considered as an option for random liver biopsy.

\section{Introduction}

Endoscopic ultrasound-guided liver biopsy (EUS-LB) is an established method for tissue acquisition of hepatic parenchyma for histologic analysis [1]. LB remains the gold standard for the di- agnosis and staging of a variety of hepatic disorders [2]. Although non-invasive testing such as serologic markers, imaging modalities, and elastography have reduced the need for LB, histologic analysis remains necessary in a variety of clinically important situations. This includes but is not limited to diagnosis 
and response to therapy in auto-immune hepatitis, AMA-negative primary biliary cholangitis, small-duct primary sclerosing cholangitis, response therapy for chronic hepatitis B infection, infiltrative disorders, and discerning simple steatosis from non-alcoholic steatohepatitis (NASH) [3].

$\mathrm{LB}$ is most commonly performed using percutaneous (PC-LB) and transjugular (TJ-LB) approaches. Limitations of the PC-LB method include sampling error as only the right lobe is accessible for biopsy, pain, hemorrhage, and hematoma. Limitations of TJ-LB approach include neck hematoma, vascular injury, arterio-venous fistula, and intra-abdominal hemorrhage [4].

EUS fine-needle aspiration (FNA) and fine-needle biopsy (FNB) have been routinely employed for targeting abnormal solid and cystic liver lesions accessible from transgastric or transduodenal approaches [5]. More recently, EUS-LB to diagnose parenchymal liver disease has been shown to be as effective as both PC-LB and TJ-LB approaches in terms of diagnostic yield [6]. In addition, EUS-LB has the advantage of sampling multiple segments of the liver. Finally, this technique has been shown to have a favorable safety profile as a recent meta-analysis found the pooled adverse event rate to be $2.3 \%$ [7].

Despite the increasing use of EUS-LB, there is a paucity of data regarding the safety and efficacy in comparison with PCLB and TJ-LB approaches. The purpose of this study was to compare the efficacy and safety profiles of EUS-LB, PC-LB, and TJ-LB.

\section{Patients and methods}

A retrospective analysis was performed at a tertiary academic medical center. The study was approved by the institutional review board and informed consent was waived given the retrospective nature of the study. Inclusion criteria were all adult patients that underwent EUS-LB since inception of this procedure at our institution (October 2013 to June 2019) and TJ-LB/PC-LB over a 3-year span (June 2016 to June 2019). Exclusion criteria included patients under 18 years old, patients with concern for primary or secondary hepatic malignancy, or if the indication for biopsy was a discrete imaging finding. The indications for hepatic biopsy were recorded. Hepatobiliary pathology records were utilized to create a patient database and they were stratified according to type of modality used for random hepatic biopsy. All biopsy specimens were assessed by expert liver pathologists.

EUS-LB was performed by five experienced interventional endoscopists. All EUS-LB were performed using a 19-gauge needle (Expect FNA 2013 to 2017, Acquire FNB 2017 to 2019 Boston Scientific). The core sample was confirmed by the therapeutic endoscopy team. The majority of specimens were obtained from the left hepatic lobe via a transgastric approach.

The primary outcome assessed was any adverse events related to EUS-LB, PC-LB, or TJ-LB. The definition of adverse event $(A E)$ was adopted from the International Organization of Standardization (ISO) and defined as "any untoward medical occurrence, unintended disease or injury, or untoward clinical signs" [8]. These were considered related to the procedure if they occurred within 30 days. Secondary outcomes included technical success defined as sufficient tissue acquisition for histologic a- nalysis and diagnostic adequacy defined as ability to reach a histopathologic diagnosis independent of number of portal triads or fragmentation. Demographic data and the indication for the procedures were all collected. The histologic findings were collected.

The statistical analysis was performed by a professional statistician in the department of public health. All measures were summarized by biopsy method using means and standard deviations for continuous measures and counts and percentages for categorical measures. Mean ages were compared between methods using an analysis of variance (ANOVA) model. Distributions of patient sex, etiology, location, technical success, diagnostic adequacy, and the occurrence of adverse events were compared between methods using either chi-square tests or Fisher's exact test, as appropriate. All analyses were performed using STATA version 16.1 (StataCorp LLC, College Station, Texas, United States).

\section{Results}

A total of 513 patients were reviewed and included for statistical analysis. There were 135 EUS-LB, 287 PC-LB, and 91 TJ-LB patients. The mean age of each group was 53 years old for EUS-LB, 52 years old for PC-LB, and 51 years old for TJ-LB. There was an overall female preponderance at $52.4 \%$. The most common indication for $L B$ regardless of modality was abnormal liver function tests (EUS-LB 89\%, PC-LB 89\%, TJ-LB 55\%). The demographic data and indications for liver biopsy are summarized in - Table 1.

For the primary outcome, the total rate of AEs was low with five reported $(<1 \%)$. There were two events in the EUS-LB group, two events in the PC-LB group, and one event in the T]LB group. This difference was not statistically significant $(P=$ $0.585)$. There was a single patient with 30 -day mortality in the EUS-LB group, however, this was determined to be unrelated to the procedure. The technical success rate was $100 \%$ in each group. The rate of diagnostic adequacy was $100 \%$ in TJ-LB group and $99 \%$ in both EUS-LB and PC-LB groups. This difference was not statistically significant $(P=1.000)$. The study outcomes are summarized in $>$ Table 2 . The most common histologic findings were non-specific histologic changes (33.7\%) and NASH (15.6\%). These results are summarized in > Table 3.

In each cohort, a random sample of specimens was reviewed by our hepato-pathologists and mean number of complete portal tracts (CPT) and specimen lengths were assessed. The mean number of CPT was higher in the EUS-LB group (19.7) than the PC-LB (17.44) and TJ-LB (10.5) groups. This difference was statistically significant $(P=0.0083)$. The mean specimen lengths were greater in the EUS-LB group $(3.47 \mathrm{~cm})$ than the $P C-L B$ $(2.92 \mathrm{~cm})$ and TJ-LB $(2.11 \mathrm{~cm})$ groups. This difference was also statistically significant $(P=0163)$. These results are summarized in Table 2. 
- Table 1 Demographic data and indication for biopsy

\begin{tabular}{|c|c|c|c|c|}
\hline Measure & $\begin{array}{l}\text { EUS } \\
(n=135)\end{array}$ & $\begin{array}{l}\text { Percutaneous } \\
(n=287)\end{array}$ & $\begin{array}{l}\text { Transjugular } \\
(n=91)\end{array}$ & $P$ value \\
\hline Age, years; mean (SD) & $53(15)$ & $52(15)$ & $51(15)$ & 0.54 \\
\hline Male; n (\%) & $49(36)$ & $145(51)$ & $50(55)$ & 0.007 \\
\hline \multicolumn{4}{|l|}{ Etiology; n (\%) } & \multirow{5}{*}{$<0.001$} \\
\hline - Abnormal LFT & $120(89)$ & $254(89)$ & $50(55)$ & \\
\hline - Abnormal Imaging & $9(7)$ & $5(2)$ & $3(3)$ & \\
\hline - Suspected adv fib Cirrhosis & $3(2)$ & $4(1)$ & $17(19)$ & \\
\hline - Follow-up of chronic condition & $3(2)$ & $22(8)$ & $21(23)$ & \\
\hline
\end{tabular}

- Table 2 Study outcomes.

\begin{tabular}{|l|c|c|c|}
\hline Measure & $\begin{array}{l}\text { EUS } \\
(\mathbf{n = 1 3 5 )}\end{array}$ & $\begin{array}{l}\text { Percutaneous } \\
(\mathbf{n = 2 8 7})\end{array}$ & $\begin{array}{l}\text { Transjugular } \\
\mathbf{( n = 9 1 )}\end{array}$ \\
\hline Adverse events; $\mathrm{n}(\%)$ & $133(99)$ & $285(99)$ & $90(99)$ \\
\hline - None & $1(1)$ & $1(0)$ & $1(1)$ \\
\hline - Severe pain & $0(0)$ & $1(0)$ & $0(0)$ \\
\hline - Bleeding & $1(1)$ & $0(0)$ & $0(0)$ \\
\hline - Mortality (within 30 days) & $2(1)$ & $2(1)$ & $1(1)$ \\
\hline Any adverse event; $n$ (\%) & $135(100)$ & $287(100)$ & $91(100)$ \\
\hline Technical success; $n$ (\%) & $134(99)$ & $284(99)$ & $91(100)$ \\
\hline Diagnostic adequacy; $n$ (\%) & $19.7(10)$ & $17.4(9)$ & $10.5(10)$ \\
\hline Complete portal tracts; $n$ & $3.47(10)$ & $2.92(9)$ & $2.11(10)$ \\
\hline Specimen length $(\mathrm{cm}) ; n$ & & 0.585 \\
\hline
\end{tabular}

\section{Discussion}

The acquisition of hepatic tissue via liver biopsy remains essential for the diagnosis, staging, and management of a variety of benign hepatic disorders $[9,10]$. Traditionally, liver biopsy has been performed percutaneously often with ultrasound guidance or by interventional radiology using a transjugular approach $[11,12]$. A recent alternative to these techniques is EUS-LB In a patient that is already undergoing an endoscopic procedure such as esophagogastroduodenoscopy or endoscopic ultrasound for a an accepted indication, EUS-LB can be performed with minimal added procedure time or risk [13].

In this study, we found that the safety profile and diagnostic adequacy is comparable between EUS-LB, PC-LB, and TJ-LB. The overall rate of $A E s$ was generally low in all procedure groups $(<1 \%)$. A single patient in each group had post-procedural pain requiring intravenous analgesia and a patient in the PC-LB group had self-limited bleeding. One patient in the EUBLB cohort experienced 30-day mortality, which was determined to be unrelated to the procedure.
Hepatic needle biopsy was first described by Paul Ehrlich in 1883 [14]. PC-LB is the most commonly used approach for tissue acquisition. The advantages include cost-effectiveness, limited equipment, and less technical skill required $[13,15]$. Conversely, disadvantages of this method are sample bias given only the right lobe is accessible and procedure related pain [16]. TJ-LB is and another commonly used modality for biopsy. Advantages of this technique include ability to perform hepatic venous pressure gradient measurements simultaneously and comparatively less complications $[17,18]$. However, TJ-LB confers higher cost than PC-LB, requires more technical skill, and is more time-consuming $[17,19]$.

Levy et al first described using EUS-LB for hepatic tissue acquisition using a disposable 19G Trucut needle (EUS-TCB, QuickCore, Wilson-Cook) [20]. In their experience FNB was comparable to FNA for diagnostic adequacy and was found to require fewer passes. In addition, there is the added benefit of not requiring on-site cytology for tissue preparation as opposed to FNA. Subsequent studies using updated devices with both 19G and 22G FNB needles have shown excellent diagnostic ade- 
$\checkmark$ Table 3 Diagnoses.

\begin{tabular}{|c|c|c|c|}
\hline Measure & $\begin{array}{l}\text { EUS } \\
(n=135)\end{array}$ & $\begin{array}{l}\text { Percutaneous } \\
(n=287)\end{array}$ & $\begin{array}{l}\text { Transjugular } \\
(n=91)\end{array}$ \\
\hline \multicolumn{4}{|l|}{ Diagnosis; n (\%) } \\
\hline - Adv fibrosis (F3)/cirrhosis & $14(10)$ & $9(3)$ & $22(24)$ \\
\hline - Non-alcoholic steatohepatitis & $29(21)$ & $43(15)$ & $8(9)$ \\
\hline - Viral hepatitis & $19(14)$ & $4(1)$ & $3(3)$ \\
\hline - Alcoholic hepatitis & $3(2)$ & $0(0)$ & $1(1)$ \\
\hline - Autoimmune hepatitis & $9(7)$ & $26(9)$ & $6(7)$ \\
\hline "Wilson's disease & $0(0)$ & $0(0)$ & $1(1)$ \\
\hline - Iron overload hemochromatosis & $2(1)$ & $4(1)$ & $0(0)$ \\
\hline - Drug induced liver injury & $9(7)$ & $8(3)$ & $4(4)$ \\
\hline - Non-specific histologic change & $41(30)$ & $97(34)$ & $35(38)$ \\
\hline - Acute or chronic rejection & $1(1)$ & $89(31)$ & $4(4)$ \\
\hline - Primary sclerosing cholangitis & $0(0)$ & $3(1)$ & $0(0)$ \\
\hline " Primary biliary cholangitis & $7(5)$ & $1(0)$ & $3(3)$ \\
\hline - Malignancy & $1(1)$ & $0(0)$ & $3(3)$ \\
\hline - Alpha-1-antitrypsin deficiency & $0(0)$ & $1(0)$ & $0(0)$ \\
\hline - Graft-versus-host disease & $0(0)$ & $1(0)$ & $1(1)$ \\
\hline
\end{tabular}

quacy and efficacy [21-23]. EUS-LB confers an advantage over other modalities of being able to obtain tissue from multiple segments of the liver, thus limiting sample error [24]. However, access to the right lobe via a transduodenal approach is technically more demanding than the left lobe via a transgastric approach [25]. Disadvantages of this approach are the cost and sedation related to the procedure. This is generally mitigated by performing this procedure in conjunction with an endoscopic procedure for another indication [21]. However, there remains a paucity of data regarding the safety profile and diagnostic adequacy of EUS-LB in comparison with other approaches.

This study has several strengths. First, this is a large cohort of EUS-LB patients in comparison with most previous studies with over 135 included for analysis. Second, EUS-LB was performed by five experienced endoscopists, thus improving generalizability. This study also has limitations. As with any retrospective study there is the potential of confounding from variables that were unable to be measured. In addition, there was incomplete documentation for the EUS-LB cohort in regard to which lobe was accessed and regarding which technique was utilized. This can be valuable information as previous studies have shown that biopsies from multiple hepatic segments may decrease sample bias [26]. All AEs were found retrospectively and thus there is the potential that they were not completely captured. Finally, there is a potential selection bias in patients that have undergone a liver transplant as these patients are un- der the care of a transplant hepatologist that may perform PCLB over other approaches.

\section{Conclusion}

In conclusion, the results of this study show that EUS-LB is associated with low rate of $A E s$ and high diagnostic adequacy in comparison with TJ-LB and PC-LB. EUS-LB should be considered as an option for acquisition of hepatic tissue for histologic analysis, especially if another endoscopic procedure is planned. Further prospective and potentially multicenter studies are warranted to validate these findings.

\section{Competing interests}

The authors declare that they have no conflict of interest.

\section{References}

[1] DeWitt J, McGreevy K, Cummings O et al. Initial experience with EUSguided Tru-cut biopsy of benign liver disease. Gastrointest Endosc 2009; 69: 535-542

[2] Bravo AA, Sheth SG, Chopra S. Liver biopsy. N Engl J Med 2001; 344 : 495-500

[3] Tapper EB, Lok AS-F. Use of liver imaging and biopsy in clinical practice. N Engl J Med 2017; 377: 756-768 
[4] Adler DG, Mengert K. EUS-guided liver biopsy: an emerging diagnostic modality. Prac Gastroenterol; https://practicalgastro.com/wpcontent/uploads/2019/09/EUS-Guided-Liver-Biopsy-Emerging-Diagnostic-Modality.pdf

[5] Hollerbach S, Willert J, Topalidis T et al. Endoscopic ultrasound-guided fine-needle aspiration biopsy of liver lesions: histological and cytological assessment. Endoscopy 2003; 35: 743-749

[6] Pineda JJ, Diehl DL, Miao CL et al. EUS-guided liver biopsy provides diagnostic samples comparable with those via the percutaneous or transjugular route. Gastrointest Endosc 2016; 83: 360-365

[7] Mohan BP, Shakhatreh M, Garg R et al. Efficacy and safety of EUSguided liver biopsy: a systematic review and meta-analysis. Gastrointest Endosc 2019; 89: 238-246.e3

[8] Ouriel K, Fowl R], Davies MG et al. Reporting standards for adverse events after medical device use in the peripheral vascular system. J Vasc Surg 2013; 58: 776-786

[9] Spycher C, Zimmermann A, Reichen J. The diagnostic value of liver biopsy. BMC Gastroenterol 2001; 1: 12-12

[10] Rockey DC, Caldwell SH, Goodman ZD et al. Liver biopsy. Hepatology 2009; 49: 1017-1044

[11] Behrens G, Ferral H. Transjugular liver biopsy. Semin Intervent Radiol 2012; 29: 111-117

[12] Ghent CN. Percutaneous liver biopsy: Reflections and refinements. Can J Gastroenterol Hepatol 2006; 20: 75-79

[13] Shah AR, Al-Hanayneh M, Chowdhry M et al. Endoscopic ultrasound guided liver biopsy for parenchymal liver disease. World J Hepatol 2019; 11: 335

[14] Moix FM, Raufman J-P. The role of liver biopsy in the evaluation of liver test abnormalities. Clin Cornerstone 2001; 3: 13-23

[15] Johnson KD, Laoveeravat P, Yee EU et al. Endoscopic ultrasound guided liver biopsy: Recent evidence. World J Gastrointest Endosc 2020; 12: 83-97
[16] Procopet B, Bureau C, Métivier S et al. Tolerance of liver biopsy in a tertiary care center: comparison of the percutaneous and the transvenous route in 143 prospectively followed patients. Eur J Gastroenterol Hepatol 2012; 24: 1209-1213

[17] Ble M, Procopet B, Miquel R et al. Transjugular liver biopsy. Clin Liver Dis 2014; 18: 767-778

[18] Gamble P, Colapinto RF, Stronell RD et al. Transjugular liver biopsy: a review of 461 biopsies. Radiology 1985; 157: 589-593

[19] Shah AR, Al-Hanayneh M, Chowdhry M et al. Endoscopic ultrasound guided liver biopsy for parenchymal liver disease. World J Hepatol 2019; 11: 335-343

[20] Levy M], Jondal ML, Clain J et al. Preliminary experience with an EUSguided trucut biopsy needle compared with EUS-guided FNA. Gastrointest Endosc 2003; 57: 101-106

[21] DiehI DL, Johal AS, Khara HS et al. Endoscopic ultrasound-guided liver biopsy: a multicenter experience. Endosc Int Open 2015; 3: E210E215

[22] Nieto J, Khaleel H, Challita Y et al. EUS-guided fine-needle core liver biopsy sampling using a novel 19-gauge needle with modified 1-pass, 1 actuation wet suction technique. Gastrointest Endosc 2018; 87: 469-475

[23] Hasan MK, Kadkhodayan K, Idrisov E et al. Endoscopic ultrasoundguided liver biopsy using a 22-G fine needle biopsy needle: a prospective study. Endoscopy 2019; 51: 818-824

[24] Mok SRS, Diehl DL. The Role of EUS in liver biopsy. Curr Gastroenterol Rep 2019; 21: 6

[25] Parekh PJ, Majithia R, Diehl DL et al. Endoscopic ultrasound-guided liver biopsy. Endosc Ultrasound 2015; 4: 85-91

[26] Regev A, Berho M, Jeffers LJ et al. Sampling error and intraobserver variation in liver biopsy in patients with chronic HCV infection. Am J Gastroenterol 2002; 97: 2614-2618 